\title{
Actividad física, acorde a sexo, en estudiantes de secundaria antes y durante del distanciamiento social por el COVID-19
}

\section{Physical activity, according to sex, in junior high school students before and during social distancing by COVID-19}

\author{
HALL-LÓPEZ, Javier Arturo ${ }^{1}$ \\ OCHOA-MARTÍNEZ, Paulina Yesica \\ ALARCÓN MEZA, Edgar Ismael ${ }^{3}$
}

\begin{abstract}
Resumen
El estudio comparo el nivel de actividad física antes y durante del distanciamiento social por el COVID19 acorde a sexo, en estudiantes de secundaria, el nivel de actividad física fue menor en mujeres que en hombres, para minimizar esta situación, el profesorado puede tomar en cuenta las directrices de igualdad de género establecidas por la Organización de las Naciones Unidas para la Educación, la Ciencia y la Cultura (UNESCO), para el logro de la calidad educativa en educación física.

Palabras claves: actividad física, educación física, secundaria, sexo.
\end{abstract}

\begin{abstract}
The study compared the level of physical activity before and during social distancing due to COVID-19 according to sex, in high school students, the level of physical activity was lower in women than in men, to minimize this situation, teachers can take taking into account the gender equality guidelines established by the United Nations Educational, Scientific and Cultural Organization (UNESCO), for the achievement of educational quality in physical education.

Keywords: physical activity, physical education, secondary, sex.
\end{abstract}

\section{Introducción}

El distanciamiento social para la prevención del contagio del COVID-19, ha limitado en la población la práctica cotidiana de actividad física (Blocken, et al., 2020), para disminuir esta problemática y que los beneficios de la actividad física para la salud se mantengan, se ha recomendado realizar actividad física en casa mediante diferentes modalidades (Chen, et al., 2020), como el ejercicio aeróbico, entrenamiento de resistencia a la fuerza con el propio peso, danza, bicicletas estacionarias y ergómetros (Hammami, et al., 2020), de igual manera apoyarse de tecnología con material audiovisual de internet y aplicaciones móviles que guíen y motiven a las

\footnotetext{
${ }^{1}$ Doctor en Enseñanza de la Actividad Física y Deporte por la Universidad de Huelva, España. y Doctor en Medicia del Deporte por la Universidad Catolina Nuestra Señora de al Asuncion, Paraguay. Profesor-Investigador de Tiempo Completo de la Facultad de Deportes Universidad Autónoma de Baja California. javierhall@uabc.edu.mx

2 Doctora en Enseñanza de la Actividad Física y Deporte por la Universidad de Huelva, España. y Doctora en Medicia del Deporte por la Universidad Catolina Nuestra Señora de al Asuncion, Paraguay. Profesora-Investigadora de Tiempo Completo de la Facultad de Deportes Universidad Autónoma de Baja California. pochoa@uabc.edu.mx

${ }^{3}$ Doctor en Fisiologia del Ejercicio por la Universidad Catolina Nuestra Señora de al Asuncion, Paraguay. Profesor-Investigador de Tiempo Completo de la Facultad de Deportes Universidad Autónoma de Baja California. eiam@uabc.edu.mx
} 
personas a realizar actividad física (Tate, et al., 2015). Aunado a esto, también se ha recomendado realizar ejercicio durante la cuarentena por las negativas consecuencias físicas y mentales del sedentarismo derivadas del aislamiento social (Jiménez-Pavón, et al., 2020).

La Organización Mundial de la Salud (OMS), establece una dedicación mínima 60 minutos diarios en actividad física con intensidad moderada a vigorosa en niños, niñas y adolescentes entre 5 a 17 años de edad, respecto a la intensidad y la obtención de los mejores beneficios de la actividad física en este grupo de edad, se ha recomendado la actividad física moderada a vigorosa en el periodo de aislamiento social (Cossio-Bolaños, 2020). Regulando la intensidad de manera gráfica para garantizar los beneficios de la actividad física (Rodríguez-Núñez, 2020), de esa manera evitar el desacondicionamiento físico durante el confinamiento por la pandemia del COVID19 (Mera, et al., 2020), y de esta manera se pudiera favorecer el aumento de enfermedades cardiovasculares asociadas al sedentarismo (Lippi, et al., 2020). En ese sentido, derivado del gasto energético y el consumo calórico en el ámbito escolar, un estudio predice un aumento de peso en los estudiantes posterior a la pandemia (Rundle, et al., 2020). El Fondo de las Naciones Unidas para la Infancia (Unicef); cuenta con un plan de acción para el retorno del estudiantado a las escuelas con acciones para la prevención y el control del COVID-19, en China, país de origen de esta pandemia, de manera específica en el ámbito de la educación física se ha realizado una guía para que el profesorado implemente estrategias didácticas que minimicen la propagación del COVID-19 (Chen, et al., 2020).

En México, una investigación referente al contexto de la cultura física identifico una menor posibilidad de participar por la población de sexo femenino en realizar actividad física deportiva (Flores Fernandez, 2019), esto se ve reflejado en las recomendaciones de actividad física establecidas por la Organización Mundial para la Salud, que en la población mexicana adolescente entre 10-14 años de edad el $12.7 \%$ de las mujeres se considera físicamente activas mientras que en los hombres de la misma edad los valores son $21.8 \%$ (Medina, et al. 2018); en el contexto educativo de secundaria se observa la misma tendencia donde los estudiantes hombres (32.9\%) participan en mayor medida en actividad física moderada a vigorosa durante al educación física que las estudiantes mujeres (22.2\%) (Hall-López, 2020). En el ámbito educativo las tecnologías de la información y comunicación han sido fundamentales para dar seguimiento a los contenidos educativos (Lorgelly \& Adler, 2020). Por lo que en este contexto tecnológico, se utilizó como herramienta docente el Google Drive para valorar a distancia (Álvarez Ferrón \& Sánchez Cañizares, 2014), la actividad física mediante el cuestionario internacional de actividad física IPAQ (Craig, et al. 2003), y comparar el nivel de actividad física antes y durante del distanciamiento social por el COVID-19 acorde a sexo, en estudiantes de secundaria.

\section{Metodología}

El presente estudio fue aprobado y registrado en la Coordinación General de Posgrado e Investigación de la Universidad Autónoma de Baja California, bajo un diseño metodológico transversal, con muestreo no probabilístico por conveniencia, siguiendo los principios éticos de investigación en seres humanos de la declaración de Helsinki (Puri, et al. 2009). Se solicitó anuencia de participación a directivos y profesores de una secundaria pública de la ciudad de Mexicali Baja California, México. En total participaron 174 estudiantes de secundaria con una edad media de $15.1 \pm 4.2$ años ( 86 hombres y 88 mujeres).

\subsection{Instrumentos y procedimientos}

Para determinar el nivel de actividad física se utilizó el formato corto versión en español del cuestionario internacional de actividad física IPAQ (Craig, et al. 2003), el cual fue aplicado a los estudiantes de secundaria, mediante la plataforma de google drive creando los reactivos del cuestionario IPAQ permitió con el que se realizaron las mediciones categóricas y continuas de actividad física realizadas en los últimos 7 días, la puntuación continua estimó el gasto energético semanal expresado en minutos MET/semana (equivalentes metabólicos), 
esto se obtuvo multiplicando el valor del gasto de energía para la actividad física de acuerdo a la frecuencia semanal (días por semana) y el tiempo en minutos (minutos por día), valorando la modalidades de caminar en una media de 3.3 MET, toda la actividad física de intensidad moderada con un valor de 4 MET y la actividad física de intensidad vigorosa se valoró en $8 \mathrm{MET}$, la puntuación clasificó en tres categorías, baja, moderada y alta.

\subsection{Análisis de los datos}

Los procedimientos estadísticos propuestos para un adecuado análisis en esta investigación fue caracterizar porcentualmente la muestra empleando estadística descriptiva, de acuerdo al sexo, antes y durante el distanciamiento social por el COVID-19, con el fin compara inter e intra grupos se realizaron pruebas de análisis de varianza (ANOVA) mixtas $2 \times 2$ (grupos x mediciones) para la variable de nivel de actividad física en estudiantes hombres y mujeres.

\section{Resultados}

La distribución porcentual del nivel de actividad física acorde a sexo en los estudiantes de secundaria antes y durante el distanciamiento social por el COVID-19, es presentada en la tabla 1.

\section{Tabla 1}

Distribuion porcentual del nivel de actividad fisica acorde a sexo en los estudiantes de secundaria antes y durante el distanciamiento social por COVID-19

\begin{tabular}{ccccc}
\hline $\begin{array}{c}\text { Nivel de Actividad } \\
\text { Física }\end{array}$ & $\begin{array}{c}\text { Antes } \\
\text { COVID-19 }\end{array}$ & $\begin{array}{c}\text { Durante } \\
\text { COVID-19 }\end{array}$ & $\begin{array}{c}\text { Antes } \\
\text { COVID-19 }\end{array}$ & $\begin{array}{c}\text { Durante } \\
\text { COVID-19 }\end{array}$ \\
\hline Baja & 35.2 & 78.6 & 41.6 & 80.2 \\
Moderada & 24.8 & 14.1 & 27.3 & 13.6 \\
Alta & 40 & 7.3 & 31.1 & 6.2 \\
\hline
\end{tabular}

Nota: Distribución porcentual del nivel de actividad fisica se determinó mediante formato corto versión en español del cuestionario internacional de actividad fisica IPAQ (Craig, et al. 2003), clasificando a los estudiantes de secundaria en tres categorias baja, moderada y alta.

La figura 1 refleja los resultados de las pruebas de análisis de varianza (ANOVA) mixtas $2 \times 2$ (grupos x mediciones) indicó que una interacción estadísticamente significativa $(p=.005)$ entre los grupos y las mediciones del gasto energético semanal de actividad física, expresado en minutos MET/semana (equivalentes metabólicos), acorde al sexo. No se encontraron cambios estadísticamente significativos entre los grupos $(p=.247)$. 
Figura 1

Gasto energetico semanal de actividad fisica expresado en MET/semana

(equivalentes metabólicos), en estudiantes de secundatia hombre y mujeres antes y durante el distanciamiento social por COVID-19

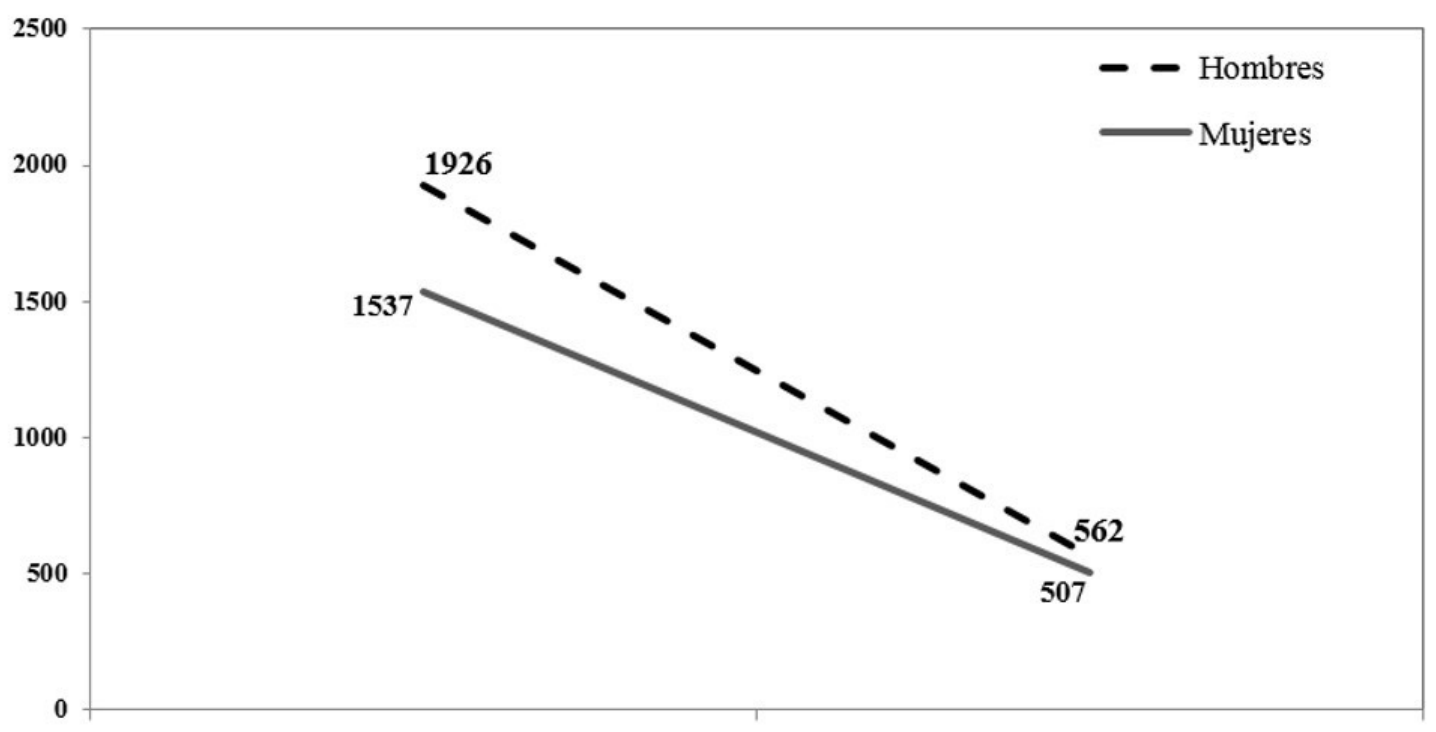

Antes COVID-19

Durante COVID-19

Nota: El gasto energético semanal expresado en minutos MET/semana (equivalentes metabólicos), esto se obtuvo del formato corto versión en español del cuestionario internacional de actividad fisica IPAQ (Craig, et al. 2003), multiplicando el valor del gasto de energia para la actividad fisica de acuerdo a la frecuencia semanal (dias por semana) y el tiempo en minutos (minutos por dia), valorando la modalidades de caminar en una media de $3.3 \mathrm{MET}$, toda la actividad fisica de intensidad moderada con un valor de 4 MET y la actividad fisica de intensidad vigorosa se valoró en 8 MET. )

El principal resultado de esta investigación fue que el nivel de actividad física fue mayor en estudiantes hombres que en mujeres antes del distanciamiento social por el COVID-19, aun sin embargo durante el distanciamiento social por el COVID-19, no se encontraron diferencias significativas en cuanto a sexo. Al comparar los porcentajes de nivel de actividad física reportados la encuesta de salud y nutrición representativa de México, que utilizo el mismo procedimiento metodológico en adolescentes, se observan valores promedio similares en las categorías baja (40.4 vs 38.4), moderada (24.4 vs 26) y alta (35.2 vs 35.5) (Medina, et al. 2018); el nivel de actividad física podríamos inferir que por las limitaciones derivadas del distanciamiento social durante el COVID-19, del estudiantado de secundaria limito la práctica de la actividad física, lo cual se ve reflejado en la figura 2. 
Figura 2

Porcentajes de diferencia $(\Delta \%)$ del nivel de actividad física, en estudiantes de secundatia hombres y mujeres, antes $\mathrm{y}$ durante el distanciamiento social por COVID-19.

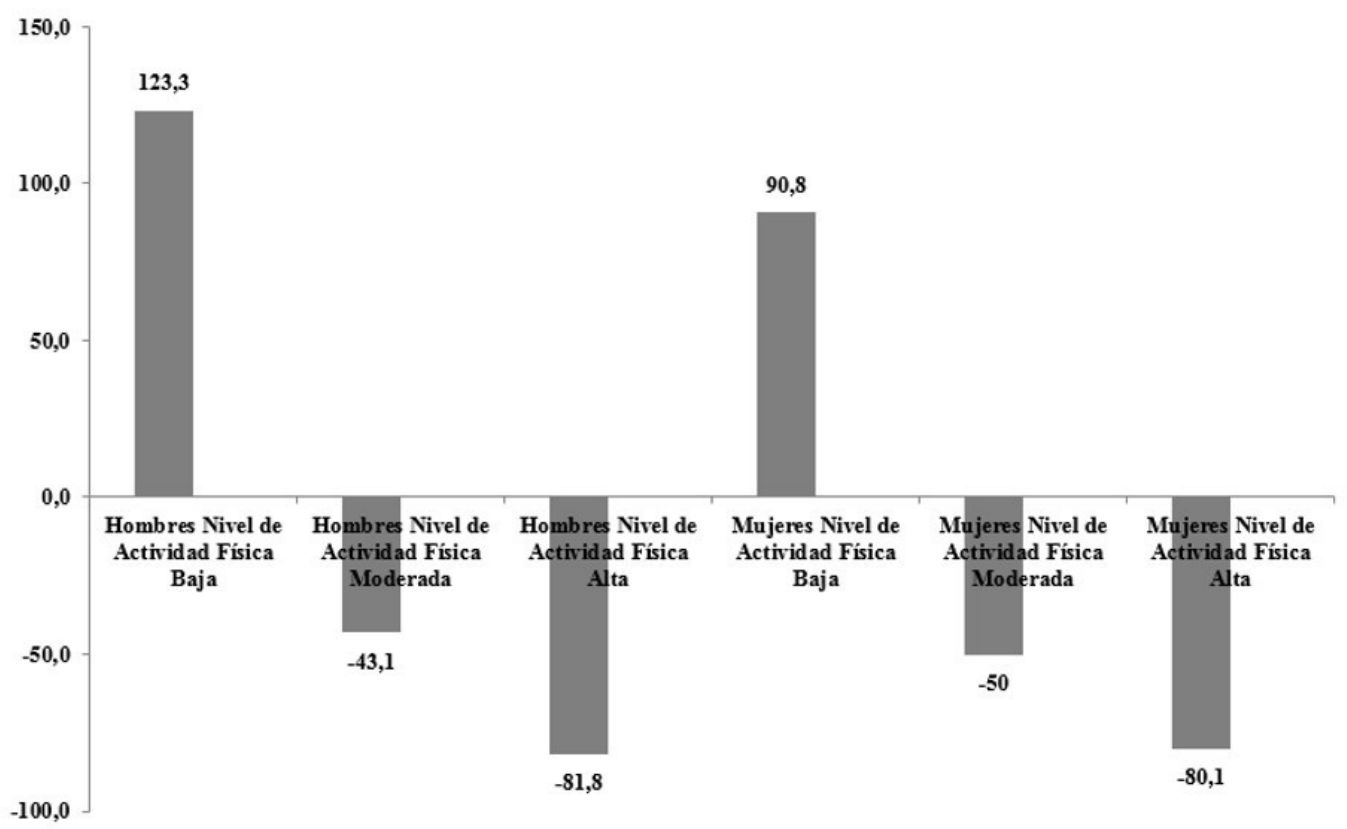

Nota: Los porcentajes de diferencia $(\Delta \%)$ de los niveles de actividad fisica clasificados como baja, moderada y alta acorde a el cuestionario internacional de actividad fisica IPAQ (Craig, et al. 2003), se determinaron mediante el siguiente procedimiento ( $\Delta \%$ ): [(Mediapost Mediapre)/Mediapre] x 100.

\subsection{Discusión}

Al identificar que previo al distanciamiento social por COVID-19, el nivel de actividad física fue menor en mujeres que en hombres es importante, que el profesorado tome en cuenta las directrices de igualdad de género establecidas por la Organización de las Naciones Unidas para la Educación, la Ciencia y la Cultura (UNESCO), para el logro de la calidad educativa en educación física (Mclennan \& Thompson, 2015), donde se manifiesta a la escuela, como un elemento clave para proporcionar a las mujeres la información, competencias y confianza necesaria para la práctica de la actividad física y deporte a lo largo de la vida (Frizzo, \& Silva Souza, 2019).

Desde el punto de vista docente la Secretaría de Educación Pública (SEP) en México establece dentro del perfil de egreso en educación secundaria, la atención al cuerpo y la salud y las habilidades digitales, en esta investigación como parte de la educación física, se utilizó como el cuestionario internacional de actividad física IPAQ (Craig, et al. 2003) y la herramienta Google Drive para valorar a distancia el nivel de actividad física (Álvarez Ferrón \& Sánchez Cañizares, 2014), lo cual es una experiencia formativa y sirve como referencia al profesorado y estudiantado de educación física en la futura implementación de estrategias pedagógicas para incrementar la actividad física posterior al COVID-19 (Chen, et al., 2020; Hall \& Ochoa-Martínez, 2020). Dadas las múltiples recomendaciones para realizar actividad física derivado de la pandemia para niños, niñas y adolescentes (CossioBolaños, 2020; Rodríguez-Núñez, 2020). Bajo las condiciones derivas de la crisis de salud en educación física, tiene un reto pedagógico y realizar actividad física en los hogares en un contexto diferente al tradicional de la escuela, donde el profesor permanentemente guía contenidos (Chen et al., 2020). 


\section{Conclusiones}

Dada la súbita emergencia sanitaria, el uso de tecnologías para identificar el nivel de actividad física del estudiantado, el rol del profesorado de educación física para la alfabetización física como prestación estructurada, que se alcanza cunado los alumnos encuentra una gama de oportunidades apropiadas en cada etapa y edad, es importante en este momento, para canalizar en el estudiante realizar actividad física durante el confinamiento social.

\section{Referencias bibliográficas}

Álvarez Ferrón, M., \& Sánchez Cañizares, L. (2014). Conocimiento, valoración y utilización, por parte del alumnado, de "Google Drive» como herramienta de trabajo cooperativo. EnseñAnza \& Teaching: Revista Interuniversitaria De Didáctica, 32(2), 23-52. doi:10.14201/et20143212352

Blocken, B., Malizia, F., van Druenen., T., \& Marchal, T. (2020). Towards aerodynamically equivalent COVID-19 $1.5 \mathrm{~m}$ social distancing for walking and running. Urban Physics, Wind Engineering \& Sports Aerodynamics. Preprint. https://www.euroga.org/system/1/user_files/files/000/045/111/45111/150d3060c/original/Social_Distan cing_v20_White_Paper.pdf

Chen, P., Mao, L., Nassis, G. P., Harmer, P., Ainsworth, B. E., \& Li, F. (2020). Coronavirus disease (COVID-19): The need to maintain regular physical activity while taking precautions. Journal of sport and health science, 9(2), 103-104. https://doi.org/10.1016/j.jshs.2020.02.001

Chen, P., Mao, L., Nassis, G. P., Harmer, P., Ainsworth, B. E., \& Li, F. (2020). Returning Chinese school-aged children and adolescents to physical activity in the wake of COVID-19: Actions and precautions. Journal of sport and health science, S2095-2546(20), 30049-1. https://doi.org/10.1016/j.jshs.2020.04.003

Cossio-Bolaños, M. (2020). Actividad física en tiempos de cuarentena por el COVID-19 en niños y adolescentes. Revista peruana de ciencias de la actividad física y del deporte, 7(2), 1-2. https://www.rpcafd.com/index.php/rpcafd/article/view/92

Craig, C. L., Marshall, A. L., Sjostrom, M., Bauman, A. E., Booth, M. L., Ainsworth, B. E., .. Oja, P. (2003). International physical activity questionnaire: 12 -country reliability and validity. Medicine \& Science in Sports \& Exercise, 35(8), 1381-1395. doi: 10.1249/01.MSS.0000078924.61453.FB

Flores Fernandez, Z. (2019). Mujer y deporte en México. Hacia una igualdad sustancial. Women and sport in México. Towards a substantive equality. Retos, 37(37), 222-226. https://recyt.fecyt.es/index.php/retos/article/view/71684

Fondo de las Naciones Unidas para la Infancia (Unicef); Key Messages and Actions for COVID-19 Prevention and Control in Schools. Disponible en: https://www.unicef.org/romania/documents/key-messages-andactions-covid-19-prevention-and-control-schools

Frizzo, G., \& Silva Souza, M. (2019). Educação física nas diretrizes da unesco: o paradigma da aptidão física e da saúde na formação do capital humano. Movimento, Porto Alegre, 25:e25022, 1-12. https://doi.org/10.22456/1982-8918.76037

Hall-López, J. (2020). Secondary physical education, participation by sex in moderate to vigorous physical activity (Educación física en secundaria, participación por sexo en actividad física moderada a vigorosa). Retos, 38(38), 543-546. https://recyt.fecyt.es/index.php/retos/article/view/77152 
Hall, J. A., y Ochoa-Martínez, P. Y. (2020). Enseñanza virtual en educación física en primaria en México y la pandemia por COVID-19. Ciencias De La Actividad Física UCM, 21(2), 1-7.

https://doi.org/10.29035/rcaf.21.2.4

Hammami, A., Harrabi, B., Mohr, M., \& Krustrup, P. (2020): Physical activity and coronavirus disease 2019 (COVID-19): specific recommendations for home-based physical training. Managing Sport and Leisure, 4:16. DOI: $10.1080 / 23750472.2020 .1757494$

Jiménez-Pavón, D., Carbonell-Baeza, A., \& Lavie, C. J. (2020). Physical exercise as therapy to fight against the mental and physical consequences of COVID-19 quarantine: Special focus in older people. Progress in cardiovascular diseases, S0033-0620(20), 30063-3. https://doi.org/10.1016/j.pcad.2020.03.009

Lippi, G., Henry, B. M., \& Sanchis-Gomar, F. (2020). Physical inactivity and cardiovascular disease at the time of coronavirus disease 2019 (COVID-19). European Journal of Preventive Cardiology, 0(0) 1-3. https://doi.org/10.1177/2047487320916823

Lorgelly, P. K., \& Adler, A. (2020). Impact of a Global Pandemic on Health Technology Assessment. Applied health economics and health policy, 7: 1-5. https://doi.org/10.1007/s40258-020-00590-9

McLennan, N. \& Thompson, J. (2015). Educación física de calidad (EFC): guía para los responsables políticos. Organización de las Naciones Unidas para la Educación, la Ciencia y la Cultura UNESCO. Disponible en: https://unesdoc.unesco.org/ark:/48223/pf0000231340

Medina, C., Jáuregui, A., Campos-Nonato, I., Barquera, S. (2018). Prevalencia y tendencias de actividad física en niños y adolescentes: resultados de Ensanut 2012 y Ensanut MC 2016. Salud Pública de México, 60(3), 263271. http://saludpublica.mx/index.php/spm/article/view/8819.

Mera, A., Tabares-Gonzalez, E., Montoya-Gonzalez, S., Muñoz-Rodriguez, D., \& Monsalve Vélez, F. (2020). Recomendaciones prácticas para evitar el desacondicionamiento físico durante el confinamiento por pandemia asociada a COVID-19. Universidad Y Salud, 22(2), 166-177.

https://doi.org/10.22267/rus.202202.188

Organización Mundial de la Salud (OMS). Recomendaciones mundiales sobre actividad física para la salud. 2010. Disponible en: https://apps.who.int/iris/bitstream/handle/10665/44441/9789243599977_spa.pdf

Puri, K. S., Suresh, K. R., Gogtay, N. J., \& Thatte, U. M. (2009). Declaration of Helsinki, 2008: Implications for stakeholders in research. Journal of Postgraduate Medicine, 55(2), 131-134. Doi 10.4103/0022-3859.52846

Rodríguez-Núñez, I. (2020). Prescribiendo ejercicio físico en períodos de cuarentena por COVID-19: ¿Es útil la autorregulación perceptual en niños?. Revista chilena de pediatría, 91(2), 304-

305. https://dx.doi.org/10.32641/rchped.vi91i2.2208

Rundle, A. G., Park, Y., Herbstman, J., Kinsey, E. W., \& Wang, Y. C. (2020) COVID-19-Related School Closings and Risk of Weight Gain Among Children. Obesity (Silver Spring), 28(6), 1008-1009.

https://doi.org/10.1002/oby.22813

Secretaria de Educación Pública (SEP) Aprendizajes Clave para la Educación Integral. Educación Física. Educación Básica. Plan y Programas de Estudio y sugerencias de evaluación. Primera edición 2017, Ciudad de México. Disponible en: https://www.aprendizajesclave.sep.gob.mx/

Tate, D. F., Lyons, E. J., \& Valle, C. G. (2015). High-tech tools for exercise motivation: use and role of technologies such as the internet, mobile applications, social media, and video games. Diabetes spectrum : a publication of the American Diabetes Association, 28(1), 45-54. https://doi.org/10.2337/diaspect.28.1.45

Esta obra está bajo una Licencia Creative Commons Attribución-NoCommercial 4.0 International

(cc) BY-NC 